\title{
Dynamic Analysis of QTLs for Green Leaf Area Duration and Green Leaf Number of Main Stem in Wheat
}

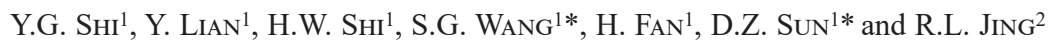 \\ ${ }^{1}$ College of Agronomy, Shanxi Agricultural University, Taigu, 030801, P.R. China \\ ${ }^{2}$ Institute of Crop Science, Chinese Academy of Agricultural Sciences, Beijing 100081, P.R. China
}

(Received 12 November 2018; Accepted 31 January 2019;

Communicated by P.S. Baenziger)

\begin{abstract}
Senescence in a wheat (Triticum aestivum L.) leaf is a programmed degeneration process leading to death. During this process, green leaf area duration (GLAD) and green leaf number of main stem (GLNMS) are gradually reduced. In this study, the two traits of Hanxuan 10 /Lumai14 DH population at different development stages after anthesis were evaluated under rainfed and irrigated conditions, and QTLs were detected. GLAD and GLNMS of two parents and DH population under rainfed condition were less than those under irrigated condition, and close correlations $(\mathrm{P}<0.05)$ were found between GLAD and GLNMS after 25 DAA under both water conditions. GLAD and GLNMS were co-controlled by major and minor genes. QTLs for GLAD were stably expressed at different development stages after anthesis under both water conditions, such as QGlad ${ }^{22}-1 B-1, Q$ Glad $^{25}-1 B-1$, Glad $^{28}-1 B-2$ detected under irrigated condition and $Q$ Glad $^{25}-1 B-3$, Glad $^{28}-1 B-4$ mapped under rainfed condition were located at a $20.7 \mathrm{cM}$ marker interval of Xgwm273-EST122 on 1B chromosome. But QTLs for GLNMS were inducibly and specifically expressed at specific developmental stages after anthesis under both water conditions. The findings provide dynamic genetic information related to wheat senescence.
\end{abstract}

Keywords: double haploid population, senescence, major gene, minor gene, correlation

\section{Introduction}

Plant leaves are either light green or dark green, due to the rich chlorophyll content (Bekavac 1998). Green colour of leaf gradually fades when the plant ages or is stressed by the environment. In turn, normal photosynthesis cannot be carried out by chlorotic leaves. The reduction of synthetic dry matter from chlorotic leaves led to a decrease in crop yield. Drought and high temperature can accelerate leaf senescence, but leaves of some cultivars (genotypes) keep green and vigorous photosynthesis under drought or high temperature. This characteristic is called 'stay-green' (Rosenow et al. 1983).

Stay-green means delayed senescence during the post-anthesis stages of plant development (Thomas 2000). It may be caused by the delayed onset of senescence or a slower rate of senescence (Thomas 1993). It was found that stay-green wheat cultivars had significantly higher photosynthetic pigment content, and can keep higher photosynthetic 
ability, furthermore was with strong drought stress, when compared with non stay-green wheat cultivar (Fotovat et al. 2007; Munné-Bosch 2000; Kang and Zhang 2004; Xin et al. 2018). Chlorophyll content and carotenoid concentration in stay-green wheat cultivars were higher, but chlorophyllase and protease activities were lower. These results explain the stay-green trait in a wheat cultivar with strong drought stress (Xue 2010). Spano et al. (2003) reported that grain weight and yield of 4 stay-green mutants were higher than those of wild types under drought condition. On the other hand, plant species or cultivars had their own senescence patterns, and difference between senescence patterns was determined genetically (Pierce et al. 1984; Walulu et al. 1994). Many reports across plant species indicated that the stay-green trait was quantitative. To date, for the percent green flag leaf area $(\%$ GFLA $+14 \mathrm{~d})$ in wheat, one QTL was detected on chromosome $2 \mathrm{~B}$ under irrigated condition, but another for \%GFLA at $+14 \mathrm{~d}$ and at $+35 \mathrm{~d}$ was identified on chromosome 2D under rainfed condition (Verma et al. 2004). Three QTLs for stay-green character were mapped on the chromosomes 1AS, 3BS and 7DS (Kumar et al. 2010). Sixteen QTLs for traits related to wheat leaf senescence were found under optimal temperature and heat stress conditions (Vijayalakshmi et al. 2010). It is well known that senescence of plant leaves is an internally programmed degeneration process, and stay-green character is closely related to the whole process of senescence (Pierce et al. 1984; Walulu et al. 1994). Obviously, it is necessary to track and investigate plant aging process for evaluating the stay-green character of plants. In this study, green leaf area duration (GLAD) and green leaf number of main stem (GLNMS) of Hanxuan10/Lumai14 DH population at different development stages after anthesis were measured under irrigated and rainfed conditions, and QTLs for these traits were identified. The purpose is to understand the dynamic behavior of quantitative trait expression related to wheat senescence, and provide more desirable QTLs for molecular marker-assisted breeding (MAB).

\section{Materials and Methods}

\section{Materials and field designs}

A wheat DH population, derived from a Hanxuan 10 (H10) $\times$ Lumai 14 (L14) cross was used as experimental material. The DH population with 150 lines was established by in vitro culture of anthers. The female parent, Hanxuan 10, is an excellent drought-resistant variety and its leaf senescence is slower during the grouting process. The male parent, Lumai 14 , is a high-yielding cultivar adapted to abundant water condition and its leaf senescence is faster during the grouting process, and the variation among various lines within the population is extensive (Jing et al. 1999). Experiment was carried out in the wheat field of Shanxi Agricultural University $\left(37^{\circ} 25^{\prime} \mathrm{N}, 112^{\circ} 25^{\prime}\right.$ E) during 2016/2017 and $2017 / 2018$. The previous crop in the experimental field was wheat. Enough moisture in the experimental field was provided by irrigation before sowing. In 2016-2017, the field design consisted of randomized complete blocks with three replications. Each plot consisted of two rows of $2 \mathrm{~m}$ long, with $25 \mathrm{~cm}$ between rows. Forty seeds were sown in 
each row. Two water regimes were used. One was rainfed with a total of $109 \mathrm{~mm}$ rainfall during the wheat growing season, the other was irrigated with a total of $650 \mathrm{~mm}$ of water applied at before winter, at seedling establishment stage, at jointing stage and at middle grain filling stage, respectively.

In 2017-2018, experiment was also carried out in field of Shanxi Agricultural University. The design of the experiment was the same as the previous year. The test materials were sown on September 25, 2017. Rainfall during the growing season was $128 \mathrm{~mm}$. Irrigation treatment was the same as the previous year.

\section{Phenotypic evaluation}

For each line and parents of DH population, ten flowering plants were tagged. Green leaf area duration and GLNMS were estimated visually. Green leaf area duration was observed from 10th day after anthesis (10DAA) to physiological maturity at 3-day intervals using a $0-9$ scale, where 0 was complete senescence and 9 was completely green over the whole leaf area (described by Joshi et al. 2007). GLNMS was recorded from 10DAA to physiological maturity at 5-day intervals, because GLNMS did not change much in three days. Green part of leaf area/total leaf area is calculated for a leaf with yellow parts.

\section{Data analysis}

The data for both the years were averaged. Using the SPSS v.19.0 statistical package, variance (ANOVA) of the data were calculated and analyzed, including the mean, standard deviation, coefficient of variation (CV), kurtosis and skewness for GLAD and GLNMS.

QTL Mapper version 2.0 software program for composite interval mapping of a mixed linear model (Wang et al. 1999) was used to detect the QTLs for GLAD and GLNMS under the two water conditions. Threshold LOD value to declare a QTL was 2.50. The QTL were designated according to the rule of 'QTL + trait + chromosome + gene number'.

\section{Results}

Variances of GLAD and GLNMS from two parents and DHLS

Under rainfed condition, GLAD of the flag leaf and GLNMS from H10 and L14 were smaller than those under irrigated. Except for GLAD at DAA10 and DAA13, the averages of GLAD of the flag leaf and GLNMS in DHLs were also smaller than those under irrigated condition, as expected. Under both water conditions, the averages of GLAD and GLNMS in DHLs were mostly between the two parents (Table 1). For GLAD and GLNMS in DHLs, the DHLs were normally distributed with wide continuous variation ranges, and transgressive segregants. 
Table 1. Phenotypic variation of green leaf area duration (GLAD) and green leaf number of main stem (GLNMS) of DH lines and their parents under rainfed and irrigated conditions

\begin{tabular}{|c|c|c|c|c|c|c|c|c|c|c|}
\hline \multirow{2}{*}{ Trait } & \multirow{2}{*}{ Treatment } & \multicolumn{2}{|c|}{ Parent } & \multicolumn{7}{|c|}{ DH lines } \\
\hline & & $\mathrm{H} 10$ & L14 & DH mean & SD & Skewness & Kurtosis & Min & Max & $\mathrm{CV}(\%)$ \\
\hline \multirow{2}{*}{$\begin{array}{l}G L A D \\
-D A A 10\end{array}$} & irrigated & 8.16 & 8.19 & 8.01 & 0.66 & -1.51 & 5.12 & 0.11 & 8.41 & 8.25 \\
\hline & rainfed & 8.14 & 8.18 & 8.07 & 0.14 & -0.44 & 0.40 & 7.67 & 8.39 & 1.75 \\
\hline \multirow{2}{*}{$\begin{array}{l}G L A D \\
-D A A 13\end{array}$} & irrigated & 8.12 & 8.10 & 7.97 & 0.66 & -1.42 & 3.60 & 0.11 & 8.34 & 8.27 \\
\hline & rainfed & 7.99 & 8.10 & 7.98 & 0.17 & -1.30 & 3.23 & 7.16 & 8.35 & 2.17 \\
\hline \multirow{2}{*}{$\begin{array}{l}G L A D \\
-D A A 16\end{array}$} & irrigated & 8.01 & 7.86 & $7.87 * *$ & 0.68 & -0.31 & 5.31 & 0.11 & 8.32 & 8.58 \\
\hline & rainfed & 7.61 & 7.86 & 7.70 & 0.42 & -2.38 & 8.05 & 5.37 & 8.26 & 5.44 \\
\hline \multirow{2}{*}{$\begin{array}{l}G L A D \\
-D A A 19\end{array}$} & irrigated & 7.67 & 7.30 & $7.60 * *$ & 0.76 & -6.86 & 2.05 & 0.11 & 8.22 & 9.99 \\
\hline & rainfed & 6.76 & 7.12 & 6.82 & 1.08 & -2.04 & 5.39 & 1.70 & 8.03 & 15.87 \\
\hline \multirow{2}{*}{$\begin{array}{l}G L A D \\
-D A A 22\end{array}$} & irrigated & 6.71 & 6.02 & $6.69 * *$ & 1.11 & -2.26 & 8.79 & 0.10 & 8.06 & 16.57 \\
\hline & rainfed & 5.01 & 5.18 & 4.69 & 1.85 & -0.76 & -0.17 & 0.00 & 7.74 & 39.42 \\
\hline \multirow{2}{*}{$\begin{array}{l}G L A D \\
-D A A 25\end{array}$} & irrigated & 4.40 & 3.67 & $4.23 * *$ & 1.99 & -0.25 & -0.82 & 0.00 & 7.82 & 47.06 \\
\hline & rainfed & 2.37 & 1.86 & 1.76 & 0.54 & 0.79 & -0.33 & 0.00 & 6.62 & 30.74 \\
\hline \multirow{2}{*}{$\begin{array}{l}G L A D \\
-D A A 28\end{array}$} & irrigated & 1.18 & 1.02 & $1.52 * *$ & 0.67 & 1.18 & 0.54 & 0.00 & 7.10 & 43.91 \\
\hline & rainfed & 0.36 & 0.07 & 0.31 & 0.08 & 3.28 & 2.12 & 0.00 & 4.26 & 25.22 \\
\hline \multirow{2}{*}{$\begin{array}{l}G L A D \\
-D A A 31\end{array}$} & irrigated & 0.02 & 0.04 & $0.32 * *$ & 0.10 & 3.86 & 7.73 & 0.00 & 5.29 & 32.59 \\
\hline & rainfed & 0.00 & 0.00 & 0.03 & 0.01 & 8.96 & 7.95 & 0.00 & 2.14 & 36.14 \\
\hline \multirow{2}{*}{$\begin{array}{l}G L N M S \\
-D A A 10\end{array}$} & irrigated & 4.18 & 3.83 & $3.93 * *$ & 0.52 & 0.18 & 1.07 & 2.50 & 5.80 & 13.22 \\
\hline & rainfed & 3.88 & 3.80 & 3.78 & 0.38 & 0.14 & 0.46 & 2.60 & 4.80 & 10.02 \\
\hline \multirow{2}{*}{$\begin{array}{l}G L N M S \\
-D A A 15\end{array}$} & irrigated & 3.73 & 3.15 & $3.54 * *$ & 0.56 & -0.82 & 0.40 & 1.60 & 4.90 & 15.80 \\
\hline & rainfed & 3.38 & 3.10 & 3.04 & 0.53 & -0.33 & 1.63 & 0.90 & 4.10 & 17.53 \\
\hline \multirow{2}{*}{$\begin{array}{l}G L N M S \\
-D A A 20\end{array}$} & irrigated & 3.00 & 2.35 & $2.60 * *$ & 0.60 & 0.24 & 0.62 & 1.00 & 4.50 & 23.01 \\
\hline & rainfed & 1.95 & 1.70 & 1.87 & 0.66 & -0.52 & 0.32 & 0.00 & 3.50 & 35.14 \\
\hline \multirow{2}{*}{$\begin{array}{l}G L N M S \\
-D A A 25\end{array}$} & irrigated & 1.30 & 0.88 & $1.18^{* *}$ & 0.73 & 0.79 & 0.69 & 0.00 & 3.90 & 61.89 \\
\hline & rainfed & 0.43 & 0.43 & 0.38 & 0.14 & 1.49 & 3.10 & 0.00 & 2.40 & 35.17 \\
\hline \multirow{2}{*}{$\begin{array}{l}G L N M S \\
-D A A 30\end{array}$} & irrigated & 0.05 & 0.06 & $0.15 * *$ & 0.05 & 2.79 & 8.60 & 0.00 & 1.70 & 34.79 \\
\hline & rainfed & 0.00 & 0.00 & 0.02 & 0.01 & 3.18 & 1.33 & 0.00 & 0.40 & 31.67 \\
\hline
\end{tabular}

*Significant $(\mathrm{p}<0.05) ; *$ highly significant $(\mathrm{p}<0.01)$.

Under both water conditions, GLAD of the two parents and DHLs slowly decreased before DAA20, but rapidly decreased from DAA20 to DAA28, then slowly decreased thereafter. But GLNMS was slowly decreased before DAA15, then rapidly decreased from DAA15 to DAA25, and slowly decreased thereafter (Fig. 1). 

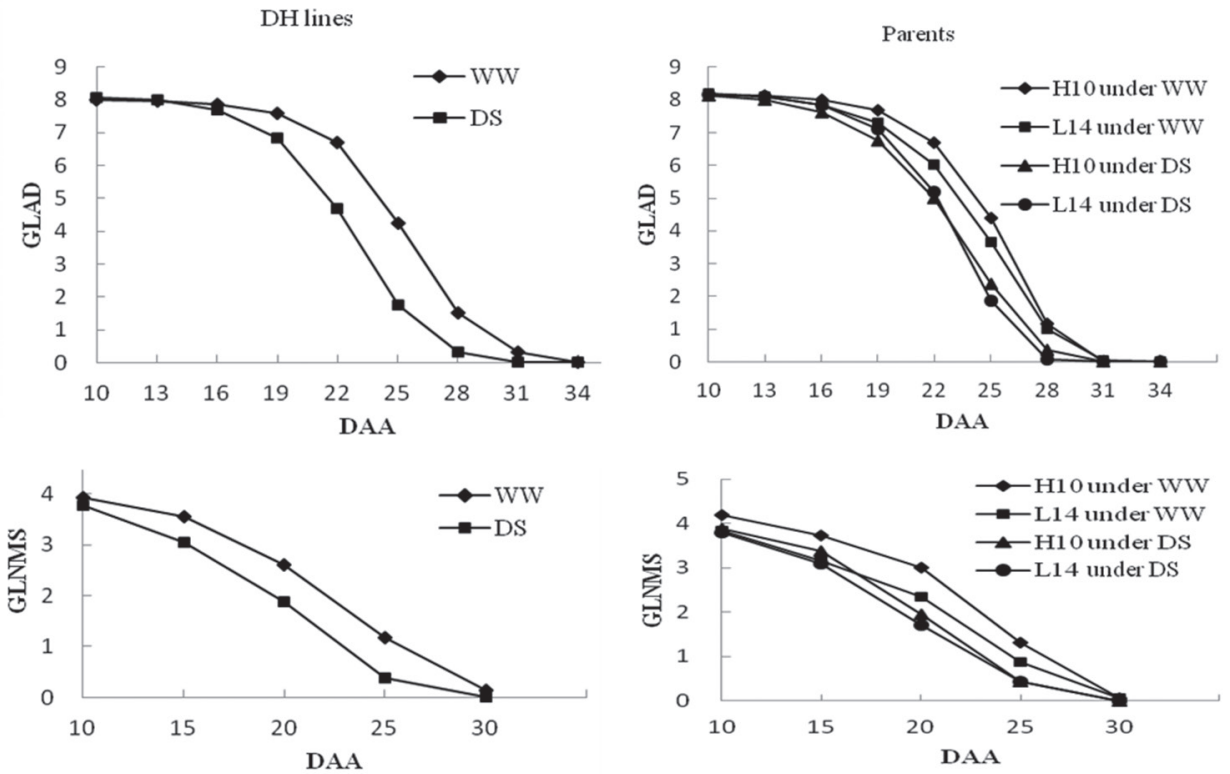

Figure 1. The variation trend of GLAD and GLNMS in DH lines and parents under the two water regimes Note: DS, drought stress; WW, well watered; DAA, days after anthesis

\section{Correlation between GLAD and GLNMS}

Under both water conditions, correlations between GLAD and GLNMS before DAA20 were not significant. But highly significant and significant correlations $(\mathrm{P}<0.05, \mathrm{P}<0.01)$ were found between GLAD-DAA22 and GLNMS-DAA15, GLNMS-DAA20 and GLNMS-DAA25, between GLAD-DAA25, GLAD-DAA28 and GLNMS at all development stages, and between GLAD-DAA31 and GLNMS-DAA20, GLNMS-DAA25 and GLNMS-DAA30 (Table 2).

\section{Dynamic QTLs for GLAD and interacting effects}

Under rainfed condition, a total of 5 additive QTLs and 6 pairs of epistatic QTLs for GLAD were detected (Tables 3, 4; Fig. 2). Phenotypic variation of these additive QTLs ranged from 8.04 to $25.56 \%$ with LOD score from 2.53 to 8.52 . These epistatic QTLs explained phenotypic variations ranging from 8.79 to $38.07 \%$ with LOD score from 5.33 to 8.64. Among these QTLs, Q Glad-5A-1was detected at DAA22 and DAA25 and with additive effects from favorable alleles of H10. QGlad ${ }^{25}-1 B-3$ located at DAA25 under rainfed condition and $Q G l a d^{28}-1 B-2$ mapped at DAA28 under irrigated condition were also at adjacent marker intervals with phenotypic variations of $18.65 \%$ and $6.05 \%$ and LOD scores from 8.52 to 2.53. All 5 additive QTLs were not involved in gene interaction. 
Table 2. Correlation coefficients between green leaf area duration (GLAD) and green leaf number of main stem (GLNMS)

\begin{tabular}{|c|c|c|c|c|c|c|}
\hline Trait & Treatment & $\begin{array}{l}\text { GLNMS } \\
\text {-DAA10 }\end{array}$ & $\begin{array}{l}\text { GLNMS } \\
\text {-DAA15 }\end{array}$ & $\begin{array}{l}\text { GLNMS } \\
\text {-DAA20 }\end{array}$ & $\begin{array}{l}\text { GLNMS } \\
\text {-DAA25 }\end{array}$ & $\begin{array}{l}\text { GLNMS } \\
\text {-DAA30 }\end{array}$ \\
\hline \multirow{2}{*}{ GLAD-DAA10 } & rainfed & 0.018 & 0.026 & 0.023 & 0.015 & 0.016 \\
\hline & irrigated & -0.094 & -0.104 & $-0.167^{*}$ & -0.18 & $-0.266 * *$ \\
\hline \multirow{2}{*}{ GLAD-DAA13 } & rainfed & 0.028 & 0.047 & 0.043 & 0.021 & 0.017 \\
\hline & irrigated & -0.085 & -0.072 & -0.138 & $-0.168 *$ & $-0.283 * *$ \\
\hline \multirow{2}{*}{ GLAD-DAA 16} & rainfed & 0.026 & 0.048 & 0.052 & 0.022 & 0.011 \\
\hline & irrigated & -0.06 & -0.003 & -0.074 & -0.127 & $-0.294 * *$ \\
\hline \multirow{2}{*}{ GLAD-DAA19 } & rainfed & 0.02 & 0.04 & 0.057 & 0.021 & 0.003 \\
\hline & irrigated & 0.007 & 0.137 & 0.078 & 0.017 & $-0.240 * *$ \\
\hline \multirow{2}{*}{ GLAD-DAA22 } & rainfed & 0.014 & 0.03 & $0.259 * *$ & $0.427 * *$ & -0.001 \\
\hline & irrigated & 0.141 & $0.318^{* *}$ & $0.353 * *$ & $0.407 * *$ & 0.016 \\
\hline \multirow{2}{*}{ GLAD-DAA25 } & rainfed & 0.021 & $0.330 *$ & $0.259 * *$ & $0.547 *$ & $0.210 *$ \\
\hline & irrigated & $0.229 * *$ & $0.311 * *$ & $0.459 * *$ & $0.734 * *$ & $0.347 * *$ \\
\hline \multirow{2}{*}{ GLAD-DAA28 } & rainfed & 0.039 & $0.241^{*}$ & $0.363^{*}$ & $0.760 * *$ & $0.334 * *$ \\
\hline & irrigated & $0.254 * *$ & $0.274 * *$ & $0.401 * *$ & $0.651 * *$ & $0.568 * *$ \\
\hline \multirow{2}{*}{ GLAD-DAA31 } & rainfed & 0.017 & 0.013 & $0.127^{*}$ & $0.327 * *$ & $0.418 * *$ \\
\hline & irrigated & 0.121 & 0.124 & $0.186^{*}$ & $0.339 * *$ & $0.526 * *$ \\
\hline
\end{tabular}

*Significant $(\mathrm{p}<0.05), * *$ highly significant $(\mathrm{p}<0.01)$

Both GLAD and GLMNS are aging-related traits, they are the result of the expression of aging-related genes in specific periods and in specific environments. The correlation between GLAD and GLMNS is a specific manifestation of pleiotropism or linkage inheritance.

Under irrigated condition, 6 additive QTLs and 3 pairs of epistatic QTLs for GLAD were detected (Tables 3, 4; Fig. 2). Phenotypic variations of these additive QTLs ranged from 5.72 to $21.10 \%$ with LOD score from 2.53 to 6.67 . Phenotypic variation of these epistatic QTLs ranged from 4.57 to $23.62 \%$ with LOD score from 5.42 to 17.46. Among them, QGlad-1B-1was mapped at DAA22 and DAA25 with the same additive effect directions. QGlad ${ }^{22}-5 A-2$ and $Q$ Glad $^{25}-5 A-3$ located at adjacent marker intervals with additive effects from favorable alleles of H10. All of 6 additive QTLs were not involved in gene interaction.

\section{Dynamic QTLs for GLNMS and interacting effects}

Under rainfed condition, 4 additive QTLs and 7 pairs of epistatic QTLs for GLNMS were detected. Phenotypic variation of these additive QTLs ranged from 10.10 to $16.99 \%$ with LOD score from 3.50 to 5.85. These epistatic QTLs explained phenotypic variation ranging from 4.91 to $20.83 \%$ with LOD score from 5.40 to 7.21 . 
$1 \mathrm{~A}$

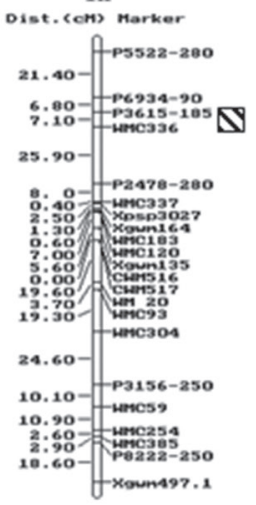

$2 A$

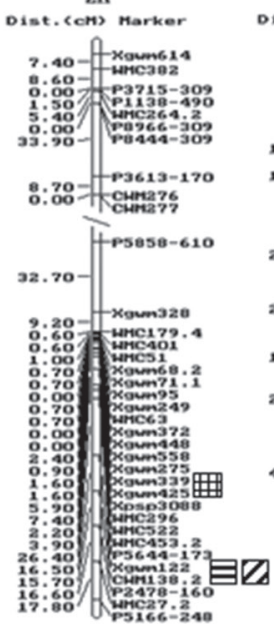

3B

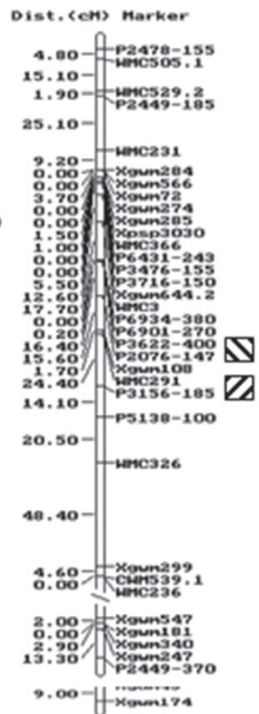

$1 \mathrm{~B}$

Dist. (c) Marke

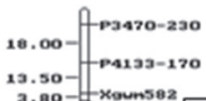

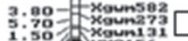

$4: 30$ gancisi

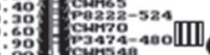

9o

oo if 951407470

:oo 103460

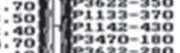

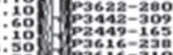

:70 $103616-310$

\%o 3 lanc269.

o: oo $10046-360$

16:00/ C4r140

7.90- $203713-309$

22. $10-$

12.60- tanc44

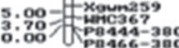

3D

Dist. (ch) Marker

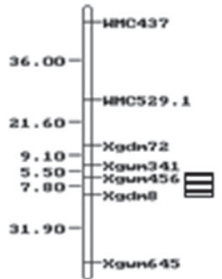

$5 A$

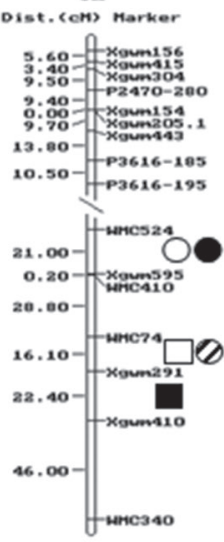

$6 A$

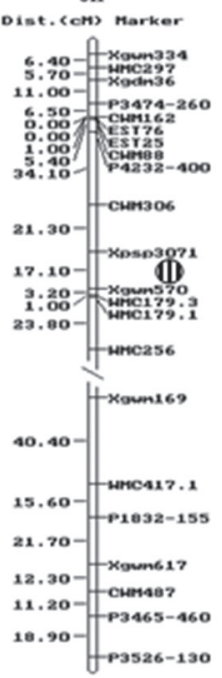

5B

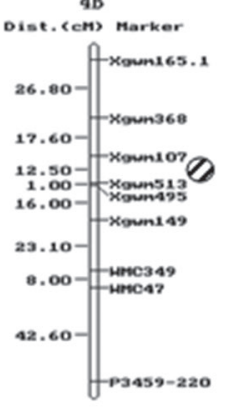

Dist. (CH) Marker

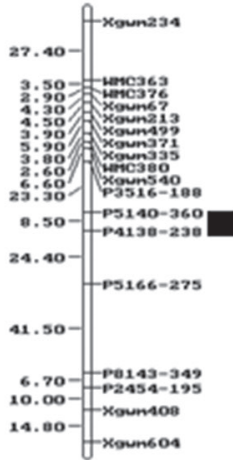

7A

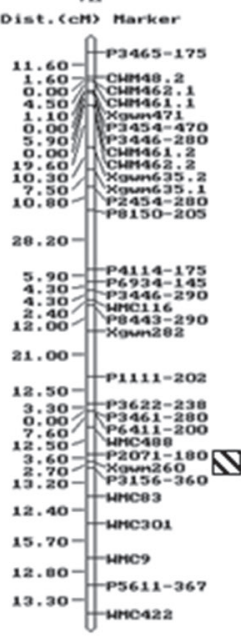

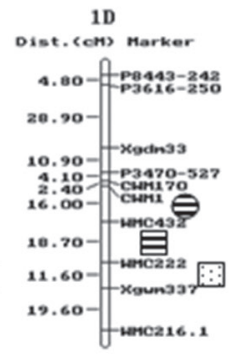

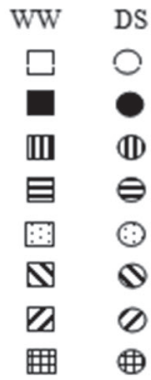

GLAD-DAA22

GLAD-DAA25

GLAD-DAA2 8

GLNMS-DAA10

GLNMS-DAA15

GLNMS-DAA20

GLNMS-DAA25

GLNMS-DAA30

Figure 2. The distribution of QTLs for GLAD and GLNMS on genetic linkage groups constructed on the DH population of 150 lines from Hanxuan 10/Lumai 
Table 3. Additive effect QTLs for green leaf area duration (GLAD) and green leaf number of main stem (GLNMS)

\begin{tabular}{|c|c|c|c|c|c|c|c|c|}
\hline Trait & Treatment & Stage & QTL & Flanking marker & $\begin{array}{c}\text { Site } \\
(\mathrm{cM})^{*}\end{array}$ & LOD & $\mathrm{AA} \dagger$ & $\mathrm{H}^{2}(\%)$ \\
\hline \multirow[t]{11}{*}{ GLAD } & \multirow[t]{6}{*}{ irrigated } & \multirow[t]{2}{*}{ DAA22 } & QGlad $^{22}-1 B-1$ & Xgwm273-Xgwm131 & 0.00 & 2.60 & -0.29 & 5.72 \\
\hline & & & QGlad $^{22}-5 A-2$ & WMC74-Xgwm291 & 0.14 & 2.88 & 0.45 & 13.76 \\
\hline & & \multirow[t]{3}{*}{ DAA25 } & QGlad $^{25}-1 B-1$ & Xgwm273-Xgwm131 & 0.04 & 6.27 & -0.73 & 11.94 \\
\hline & & & QGlad $^{25}-5 A-3$ & Xgwm291-Xgwm410 & 0.00 & 6.55 & 0.97 & 21.10 \\
\hline & & & QGlad $^{25}-5 B$ & P5140.2-P4138 & 0.02 & 6.67 & -0.93 & 19.43 \\
\hline & & DAA28 & QGlad $^{28}-1 B-2$ & $C W M 70-P 3474.4$ & 0.00 & 2.53 & -0.44 & 6.05 \\
\hline & \multirow[t]{5}{*}{ rainfed } & DAA22 & QGlad $^{22}-5 A-1$ & WMC524-Xgwm595 & 0.08 & 4.90 & 0.73 & 15.79 \\
\hline & & \multirow[t]{2}{*}{ DAA25 } & QGlad ${ }^{25}-1 B-3$ & P3474.4-CWM548 & 0.00 & 8.52 & -0.68 & 18.65 \\
\hline & & & QGlad $^{25}-5 A-1$ & WMC524-Xgwm595 & 0.04 & 7.55 & 0.67 & 18.21 \\
\hline & & \multirow[t]{2}{*}{ DAA28 } & QGlad $^{28}-1 B-4$ & P5140.3-EST122 & 0.00 & 5.41 & -0.26 & 25.56 \\
\hline & & & QGlad $^{28}-6 A$ & Xpsp3071-Xgwm570 & 0.02 & 2.53 & 0.14 & 8.04 \\
\hline \multirow[t]{15}{*}{ GLNMS } & \multirow[t]{11}{*}{ irrigated } & \multirow[t]{3}{*}{ DAA10 } & $Q G l n m s^{10}-1 D-1$ & $W M C 432-W M C 222$ & 0.02 & 6.70 & 0.20 & 16.68 \\
\hline & & & $Q G \operatorname{lnm} s^{10}-2 A-3$ & Xgwm122-CWM138.2 & 0.00 & 2.51 & -0.11 & 5.18 \\
\hline & & & $Q G \operatorname{lnm} s^{10}-3 D$ & Xgwm456-Xgdm8 & 0.00 & 2.65 & -0.12 & 5.73 \\
\hline & & DAA 15 & $Q G \operatorname{lnm} s^{15}-1 D-1$ & $W M C 432-W M C 222$ & 0.08 & 3.11 & $0.16^{*}$ & 9.92 \\
\hline & & \multirow[t]{4}{*}{ DAA20 } & $Q G \operatorname{lnm} s^{20}-1 A-1$ & P3615.2-WMC336 & 0.00 & 2.85 & 0.15 & 7.52 \\
\hline & & & $Q G \operatorname{lnm} s^{20}-3 B-1$ & P3622.4-P2076 & 0.16 & 4.08 & -0.19 & 11.07 \\
\hline & & & $Q G \operatorname{lnm} s^{20}-5 D$ & $X g d m 3-X g d m 43$ & 0.42 & 3.76 & -0.16 & 8.69 \\
\hline & & & $Q G \operatorname{lnm} s^{20}-7 A$ & P2071-Xgwm260 & 0.02 & 4.54 & 0.18 & 10.47 \\
\hline & & \multirow[t]{2}{*}{ DAA25 } & $Q G \operatorname{lnm} s^{25}-2 A-2$ & P5644.1-Xgwm122 & 0.20 & 3.03 & -0.21 & 8.91 \\
\hline & & & $Q G \operatorname{lnm} s^{25}-3 B-2$ & WMC291-P3156.1 & 0.06 & 5.53 & -0.36 & 25.70 \\
\hline & & DAA30 & $Q G \operatorname{lnm} s^{30}-2 A-1$ & Xgwm339-Xgwm425 & 0.00 & 3.87 & -0.18 & 34.82 \\
\hline & \multirow[t]{4}{*}{ rainfed } & \multirow[t]{2}{*}{ DAA10 } & $Q G \operatorname{lnm} s^{10}-1 D-2$ & $C W M 1-W M C 432$ & 0.12 & 5.85 & 0.15 & 16.99 \\
\hline & & & $Q G \operatorname{lnm} s^{10}-2 D$ & P3470.3-P3176.1 & 0.06 & 3.81 & 0.12 & 10.30 \\
\hline & & \multirow[t]{2}{*}{ DAA25 } & $Q G \operatorname{lnm} s^{25}-4 B-1$ & Xgwm107-Xgwm513 & 0.12 & 4.13 & -0.13 & 10.10 \\
\hline & & & $Q G \operatorname{lnm} s^{25}-5 A$ & WMC74-Xgwm 291 & 0.00 & 3.50 & 0.15 & 12.68 \\
\hline
\end{tabular}

*Genetic distance of the putative QTL from the left flanking marker.

$\dagger$ Additive effect, a positive value indicates the allele is from Hanxuan 10, while a negative value manes the allele is from Lumai 14 . \$The phenotypic variance explained by the additive QTL.

Eleven additive QTLs and 6 pairs of epistatic QTLs for GLNMS were detected under irrigated condition, Phenotypic variation of these additive QTLs ranged from 5.18 to $34.82 \%$ with LOD score from 2.51 to 6.70 . These epistatic QTLs explained phenotypic variations ranging from 9.55 to $22.16 \%$ with LOD score from 5.05 to 6.93. QGlnms ${ }^{10_{-}}$ $1 D-1$ and $Q G \operatorname{lnm} s^{15}-1 D-1$ detected under irrigated and $Q G l n m s^{10}-1 D-2$ mapped under 


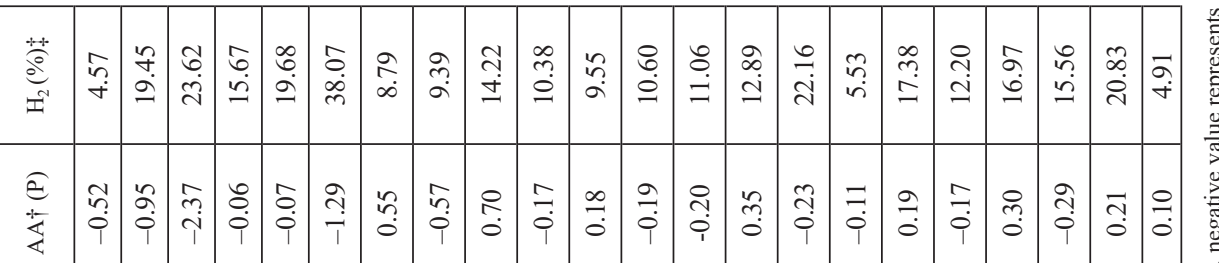

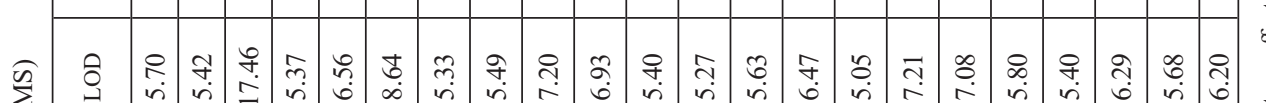

可

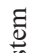

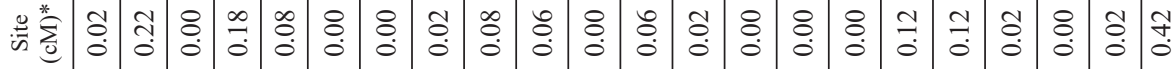

灵

$m$ m $m$ m

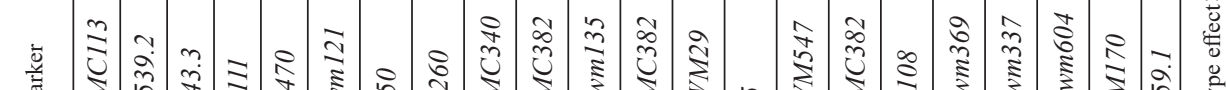

है

苟

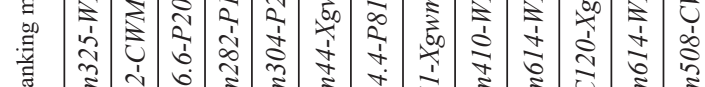

壱

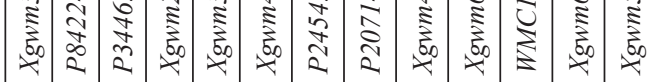

ก

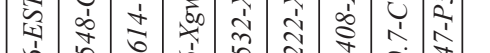

ฮั

चี

宅

已

胥

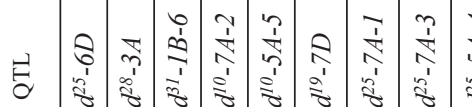

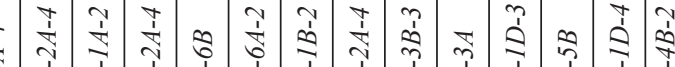

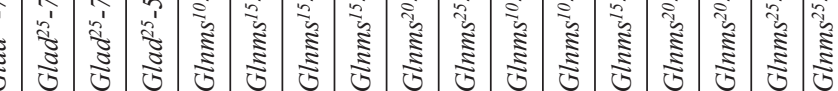

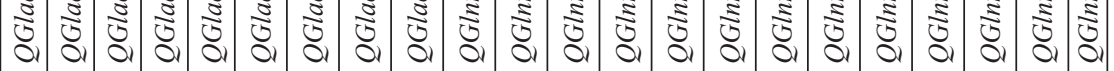

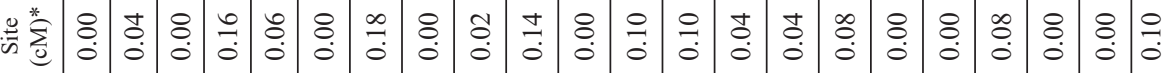

\begin{tabular}{|c|c|c|c|c|c|c|c|c|c|c|c|c|c|c|c|c|c|c|c|}
\hline 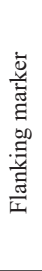 & 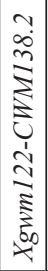 & 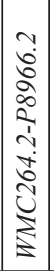 & 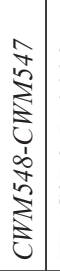 & 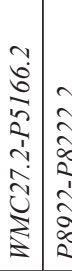 & 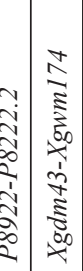 & 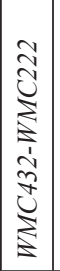 & 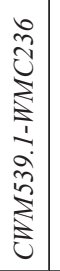 & 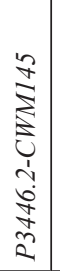 & 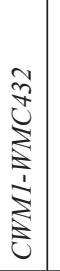 & 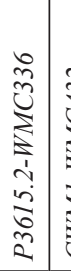 & 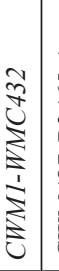 & 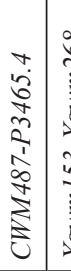 & 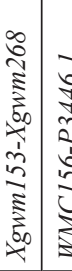 & 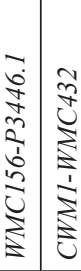 & 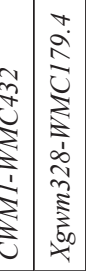 & 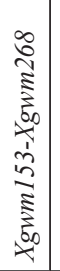 & 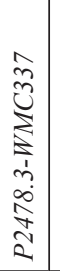 & 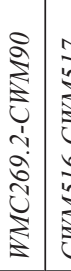 & 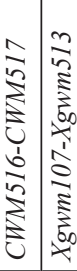 \\
\hline$\vec{\sigma}$ & 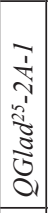 & 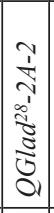 & $\begin{array}{c}n \\
0 \\
0 \\
1 \\
0 \\
\vdots \\
0 \\
0 \\
0\end{array}$ & 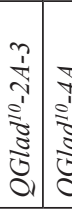 & 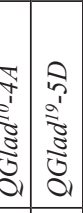 & 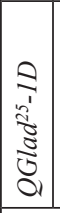 & $\begin{array}{l}0 \\
m \\
n \\
\tilde{\Xi} \\
\vdots \\
\vdots \\
\vdots\end{array}$ & 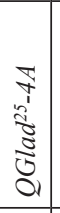 & 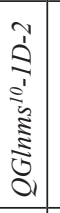 & 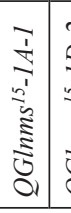 & 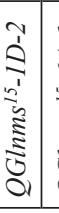 & 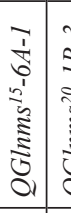 & 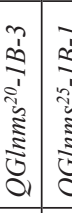 & 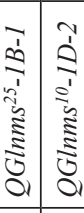 & 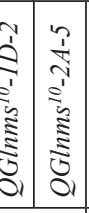 & $\begin{array}{c}n \\
0 \\
0 \\
n \\
\vdots \\
\vdots \\
\vdots \\
0 \\
0 \\
0\end{array}$ & 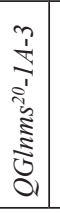 & 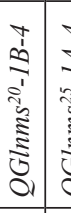 & 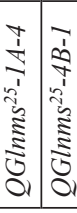 \\
\hline 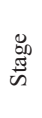 & 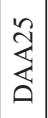 & 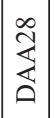 & 交 & $\stackrel{\circ}{\stackrel{4}{4}}$ & 离 & & 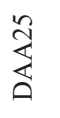 & & 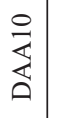 & & 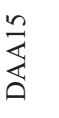 & & 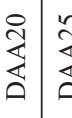 & 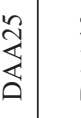 & 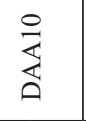 & $\begin{array}{l}n \\
\sum_{1} \\
0\end{array}$ & 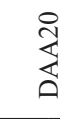 & & 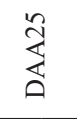 \\
\hline 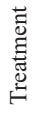 & 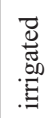 & & & 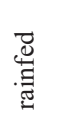 & & & & & 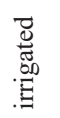 & & & & & & 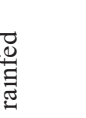 & & & & \\
\hline 营 & 窎 & & & & & & & & $\sum_{3}^{\infty}$ & & & & & & & & & & \\
\hline
\end{tabular}


rainfed were near the marker Xwmc432 on chromosome 1D and with additive effects from favorable alleles of H10. In addition, QGlnms ${ }^{10}-1 D-2, Q G \ln m s^{20}-1 A-1$ and $Q G \operatorname{lnm} s^{25}-4 B-1$ were all involved in gene interaction (Tables 3, 4; Fig. 2).

\section{Discussion}

Variation of GLAD and GLNMS in wheat

Under irrigated condition, GLAD and GLNMS of H10 were higher than those of L14 during the whole senescence process. Under rainfed condition, when compared with L14, GLAD of H10 was higher from flowering (0 DAA) to 19 DAA, a little smaller after 20 DAA. But GLNMS of H10 was more from flowering (0 DAA) to 25 DAA, then tended to the same. This suggested water condition had different effects on GLAD and GLNMS of the two parents.

The average values of GLAD and GLNMS in DHLs under rainfed condition were smaller than those under irrigated condition, except for GLAD at DAA10 and DAA13, suggesting drought stress accelerated wheat leaf senescence, especially during middle and late grain filling stages. Verma et al. (2004) also found that drought stress decreased chlorophyll content of wheat leaves, resulted in premature senescence of wheat leaves, and a decrease of the number of green leaves. Collectively, these effects reduced the capacity of leaf assimilation and reduced yield. Therefore, irrigation is helpful to ensure a sufficient soil moisture content for delaying wheat leaves senescence. It was previously found that a line or cultivar with a higher maximum rate of senescence often had a later onset time of leaf senescence (Wang et al. 2015). This may be a self-regulating mechanism of the plant itself to optimize senescence.

\section{Genetic effect of GLAD and GLNMS}

Tanksley and Nelson (1996) thought that a QTL explained more than 10 percentage of phenotypic variations was main effect. In this study, phenotypic variation explained by 8 of 11 additive QTLs for GLAD were more than 10\%, and phenotypic variation explained by 6 pairs of 9 pairs epistatic QTLs for GLAD were more than 10\%, while phenotypic variation explained by 9 of 15 additive QTLs for GLNMS were more than 10\%, and phenotypic variation explained by 10 pairs of 13 pairs epistatic QTLs for GLNMS were more than $10 \%$. As some of the QTLs for both traits mapped to the same region, it appears GLAD and GLNMS in wheat were co-controlled by many of the same major and minor genes.

$\mathrm{Wu}$ (2008) observed chlorophyll content at flowering stage was controlled by additive effect genes, while controlled by epistatic effect genes at grain filling stage. But Shen et al. (2005) showed chlorophyll content was related to additive, epistatic, additive environment interaction and epistatic environment interaction effects. In the study, 3 (QGlnms ${ }^{10_{-}}$ $1 D-2, Q G \operatorname{lnm} s^{20}-1 A-1$ and $\left.Q G l n m s^{25}-4 B-1\right)$ of 15 additive QTLs for GLNMS were in- 
volved in gene interaction, but none of 11 additive QTLs for GLAD exhibited gene interactions, suggesting complexity of genetic systems related to senescence in wheat leaves.

\section{Pleiotropy or linked genetics of QTLs related to stay-green or senescence}

Many studies have showed that QTLs for closely correlated traits may be located at, or near, the same chromosomal positions (Hervé et al. 2001; Fracheboud et al. 2002; Tuberosa et al. 2002). In this study, QGlad ${ }^{22}-1 B-1$, QGlad $^{25}-1 B-1$ and QGlad ${ }^{28}-1 B-2$ detected under irrigated, and $Q$ Glad $^{25}-1 B-3, Q_{\text {Glad }}{ }^{28}-1 B-4$ mapped under rainfed were located at a $20.7 \mathrm{cM}$ marker region Xgwm273-EST122. QTmrs-1B-1, and QTmrs-1B-2 for time to maximum rate of senescence detected by Wang et al. (2015) also were at this marker region. $Q G l n m s^{20}-1 A-1$ detected under irrigated was also at the same marker region as $Q T m r s-1 A$ for time to maximum rate of senescence, $Q T s-1 A$ for onset time of leaf senescence, $Q T o-1 A-1$ for end time of leaf senescence, and $Q 25 \% G-1 A$ for the time to reach 75\% senescence (Wang et al. 2015). QChlc.cgb-1A for chlorophyll content (Yang et al. 2007) mapped under drought stress was also at this region. On the other hand, many QTLs related to stay-green, including QGlad ${ }^{22}-5 A-2, Q_{\text {Glad }}^{25}-5 A-3, Q 75 \% G-5 A-2$ (Wang et al. 2015), QChlc.cgb-5A-2 (Yang et al. 2007) detected under well-watered, and QGlnms ${ }^{25}-5 A$, QTmrs-5A, QTo-5, Q25\%G-5A (Wang et al. 2015) mapped under drought stress were at a linked marker region WMC410-WMC74-Xgwm291-Xgwm410 on chromosome 5A. QGlad ${ }^{22}-5 A-1, Q_{\text {Glad }}^{25}-5 A-1, Q T s-5 A$ and $Q 75 \% G-5 A-1$ mapped under drought stress (Wang et al. 2015) were at another marker region Xwmc524-Xgwm595 on chromosome 5A. Therefore, four hot-spot regions related to stay-green or senescence were located on chromosomes 1A, 1B and 5A (2 regions). These QTLs may be pleiotropic (with different functions) or are tightly linked genes. One way to further understand them could be to improve the genetic map by increasing the number markers or number of progenies (Tuberosa et al. 2002). Another could be to generate inbred lines with the separate regions of interest for fine mapping to improve the rate of MAB.

\section{Effect of water condition on QTL expression}

A quantitative trait is generally controlled by multiple genes and easily influenced by environment. Hu et al. (2006) found significant effect of different environments on QTL expression, when the analysis of QTLs for chlorophyll content in rice (Oryza sativa L.) under drought stress and well-watered. Xu et al. (2000) thought QTL expression for chlorophyll content in sorghum (Sorghum bicolor L.) was also influenced by water conditions. In this study, six QTLs for GLAD and 11 for GLNMS were detected under irrigated, and 5 QTLs for GLAD and 4 for GLNMS were detected under rainfed. Among them, $Q G \operatorname{lnm} s^{10}-1 D$ was mapped under both water conditions, and was thus expressed independently of water status at the specific growing stage. In contrast, QGlad ${ }^{22}-1 B-1$ and QGlad $^{25}-1 B-1$ in the marker region Xgwm273-Xgwm131, QGlad ${ }^{22}-1 B$-1 and QGlad ${ }^{25}$ $1 B-1$ between WMC432 and WMC222, QGlad ${ }^{22}-5 A-2$ and QGlad ${ }^{25}-5 A-3$ in the marker region WMC74-Xgwm291-Xgwm410 were all detected only in irrigated trials. While 
QGlad $^{22}-5 A-2$ and QGlad $^{25}-5 A-3$ were mapped only in rainfed trials. These results showed all of these QTLs were stably expressed under the specific water condition. The rest 17 QTLs were detected under one water condition and at a specific growing stage.

\section{Stay green and resistance to drought stress in wheat}

Genotypes with stay-green character were resistant to drought (Rosenow et al. 1983; Spano et al. 2003). Previous reports indicated that drought stress induced premature leaf senescence (Buchanan-Wollaston 1997; Nooden et al. 1997), and significant differences of flag leaf senescence were found in cereal crops, such as common wheat (Verma et al. 2004; Mariana et al. 2016), sorghum (Rosenow et al. 1981), maize (Zea mays L.) (Bänziger et al. 1999) and durum wheat (Hafsi et al. 2000). It is well known that water use efficiency is one of the main physiological traits related to drought resistance in plants. QGlad $^{28}-1 B-4$ detected in this study, QTmrs-1B-2 reported by Wang et al. (2015) and $Q W c . c g b-1 B$ for water consumed mapped by Zhou et al. (2005) were all linked with marker EST122. Similarly, QGlad ${ }^{22}-5 A-1$ and QGlad $^{25}-5 A-1$ detected in this study, Q75\%G-5A-1 and QTS-5A reported by Wang et al. (2015) and QUgwue.cgb-5A for upground water use efficiency (Zhou et al. 2005) were linked with marker Xgwm595. The collocations of these QTLs demonstrated the similar genetic mechanism of stay green and resistance to drought stress in wheat.

\section{Acknowledgements}

This work was supported by National Natural Science Foundation of China (31671607) and National Science and Technology Major Projects for Cultivation of New Transgenic Cultivars (2014ZX0800203B-003), National Key R\&D Program of China (2017YFD0300202), and Key R\&D Program in Shanxi (201703D211007-6), and Key R\&D Program Projects in Shanxi (201803D221008-3).

\section{References}

Bänziger, M., Edmeades, G.O., Lafitte, H.R. 1999. Selection for drought tolerance increases maize yields across a range of nitrogen levels. Crop Sci. 39:1035-1040.

Bekavac, G. 1998. Path analysis of stay-green trait in maize. Cereal Res. Commun. 26:161-167.

Buchanan-Wollaston, V. 1997. The molecular biology of leaf senescence. J. Exp. Bot. 48:181-199.

Fotovat, R., Valizadeh, M., Toorchi, M. 2007. Association between water-use efficiency components and total chlorophyll content (SPAD) in wheat (Triticum aestivum L.) under well-watered and drought stress conditions. Int. J. Food Agric. and Enviorn. 5:225-227.

Fracheboud, Y., Ribaut, J.M., Vargas, M., Mesamer, R. 2002. Identification of quantitative trait loci for cold tolerance of photosynthesis in maize (Zea mays L.). J. Exp. Bot. 53:1967-1977.

Hafsi, M., Mechmeche, W., Bouamama, L., Djekoune, A. 2000. Flag leaf senescence as evaluated by numerical image analysis and its relationship with yield under drought in durum wheat. J. Agron. Crop Sci. 185:275280.

Hervé, D., Francoise, F., Ericka, F.B., Nadia, L. 2001. QTL analysis of photosynthesis and water status traits in sunflower (Helianthus annuus L.) under greenhouse condition. J. Exp. Bot. 52:1857-1864. 
Hu, S., Mei, H., Zou, G., Liu, H. 2006. Analysis of quantitative trait loci for chlorophyll content in rice leaves under drought stress. J. Plant Ecol. 30:479-486.

Jing, R.L., Chang, X.P., Jia, J.Z., Hu, R.H. 1999. Establishing wheat doubled haploid population for genetic mapping by anther culture. Biotechnol. 9:4-8.

Joshi, A.K., Kumari, M., Singh, V.P., Reddy, C.M. 2007. Stay green trait: variation, inheritance and its association with spot blotch resistance in spring wheat. Euphytica 153:59-71.

Kang, S., Zhang, J. 2004. Controlled alternate partial root-zone irrigation, its physiological consequences and impact on water use efficiency. J. Exp. Bot. 55:1-10.

Kumar, U., Joshi, A.K., Kumari, M., Paliwal, R. 2010. Identification of QTLs for stay green trait in wheat (Triticum aestivum L.) in the 'Chirya 3'×'Sonalika' population. Euphytica 174:437-445.

Mariana, L., Checovich, Andrea Galatro, Jorge, I. Moriconi. 2016. The stay-green phenotype of TaNAM-RNAi wheat plants is associated with maintenance of chloroplast structure and high enzymatic antioxidant activity. Plant Physiol. Bioch. 104:257-265.

Munné-Bosch, S., Alegre, L. 2000. Changes in carotenoids, tocopherols and diterpenes during drought and recovery and the biological significance of chlorophyll loss in Rosmarinus officinalis plants. Planta 210:925-931.

Nooden, L.D., Guiamet, J.J., John, I. 1997. Senescence mechanisms. Physiol. Plant. 101:746-753.

Pierce, R.O., Knowles, P.F., Phillips, D.A. 1984. Inheritance of delayed leaf senescence in soybean. Crop Sci. 24:515-518.

Rosenow, D.T., Clark, L.E. 1981. Drought tolerance in sorghum. In Proceedings of the 36th Annual Corn and Sorghum Research Conference, Held at Chicago (eds) H.D. Loden, D. Wilkinson), pp. 18-30. Washington, DC: American Seed Trade Association.

Rosenow, D.T., Quisenberry, J.E., Wendt, C.W., Clark, L.E. 1983. Drought tolerant sorghum and cotton germplasm. Agr. Water Manage. 7:207-222.

Shen, B., Zhuang, J., Zhang, K., Dai, W. 2005. Analysis of interaction between QTL and environment on chlorophyll contents in rice. Scientia Agricultura Sinica 38:1937-1943.

Spano, G., Fonzo, N.Di., Perrotta, C., Platani, C. 2003. Physiological characterization of 'stay green' mutants in durum wheat. J. Exp. Bot. 54:1415-1420.

Tanksley, S.D., Nelson, J.C. 1996. Advanced backcross QTL analysis: a method for the simultaneous discovery and transfer of valuable QTLs from unadapted germplasm into elite breeding lines. Theor. Appl. Genet. 92:191-203.

Thomas, H., Howarth, C.J. 2000. Five ways to stay green. J. Exp. Bot. 51:329-337.

Thomas, H., Smart, C.M. 1993. Crops that stay green. Ann. Appl. Biol. 123:193-219.

Tuberosa, R., Salvi, S., Sanguineti, M.C., Landi, P. 2002. Mapping QTL regulating morpho-physiological traits and yield: Case studies, shortcomings and perspectives in drought stressed maize. Ann. Bot. 89:941-963.

Verma, V., Foulkes, M.J., Worland, A.J., Sylvesterbradley. 2004. Mapping quantitative trait loci for flag leaf senescence as a yield determinant in winter wheat under optimal and drought stressed environments. Euphytica 135:255-263.

Vijayalakshmi, K., Fritz, A.K., Paulsen, G.M., Bai, G. 2010. Modeling and mapping QTL for senescencerelated traits in winter wheat under high temperature. Mol. Breed. 26:163-175.

Walulu, R.S., Rosenow, D.T., Wester, D.B., Nguyen, H.T. 1994. Inheritance of the stay-green trait in sorghum. Crop Sci. 34:970-972.

Wang, D.L., Zhu, J., Li, Z.K., Paterson, A.H. 1999. Mapping QTLs with epistatic effects and QTL $\times$ environment interactions by mixed linear model approaches. Theor. Appl. Genet. 99:1255-1264.

Wang, S., Liang, Z., Sun, D., Dong, F. 2015. Quantitative trait loci mapping for traits related to the progression of wheat flag leaf senescence. J. Agric. Sci. 153:1234-1245.

Wu, X. 2008. Genetic analysis of quantitative trait loci for drought resistance in Wheat. Chinese Academy of Agricultural Sciences. PhD thesis, Beijing, China.

Xin, X.X., Li, M.F., Liu, Y. 2018. Genetic analysis of stay-green of flag leaf and thousand-grain weight in introgression lines of wheat under different water conditions. Agric. Res. Arid. Areas. 36:207-212.

Xu, W., Subudhi, P.K., Crasta, O.R., Rosenow, D.T. 2000. Molecular mapping of QTLs conferring stay-green in grain sorghum. Genome 43:461-469. 
Xue, H. 2010. Studies on the changes of endogenous hormones in stay-green wheat varieties and the relationship between the changes of endogenous hormones and stay-green and drought resistance. Master's thesis, Northwest Agriculture and Forest Science and Technology University, Shaanxi, China.

Yang, D., Jing, R., Chang, X., Li, W. 2007. Quantitative trait loci mapping for chlorophyll fluorescence and associated traits in wheat. J. Integr. Plant Biol. 49:646-654.

Zhou, X., Jing, R., Chang, X., Zhang, Z. 2005. QTL mapping for water use efficiency and related traits in wheat seedling. J. Plant Genet. Resour. 6:20-25. 